\section{Psychiatric problems common in chronic disabling occupational spinal disorders}

The prevalence of psychiatric disorders is highly elevated in patients with chronic disabling occupational spinal disorders (CDOSDs), reports a recent study. Without appropriate intervention, the rehabilitation of these patients could be hindered.

Dersh et al. administered two versions of the Structured Clinical Interview for the Diagnostic and Statistical Manual of Mental Disorders, SCID-NP and SCID-II, to what they report to be the largest studied cohort of CDOSD patients $(n=1,323)$ presenting for a functional restoration program of rehabilitation. The site of injury in these patients was varied. Compared with the general population, patients were over 10 times more likely to have a major current DSM Axis I psychiatric disorder (64.9\% vs $15.4 \%$; especially major depressive disorder, drug abuse/dependence and anxiety disorders) and over 13 times more likely to have a major DSM Axis II personality disorder $(69.6 \%$ vs $14.8 \%$; most commonly paranoid personality disorder and borderline personality disorder). No strong association was found between psychopathology and site of spinal injury claim.

Dersh et al. recommend that clinicians treating patients with CDOSDs should be ready and willing to use the experience of mental health professionals to aid them in identifying and stabilizing those patients with psychiatric disorders. They are carrying out additional investigations to determine whether it is the pain condition or the psychopathology that occurs first; resolution of this issue will aid further study of the association between psychopathology and chronic pain.

Original article Dersh J et al. (2006) Prevalence of psychiatric disorders in patients with chronic disabling occupational spinal disorders. Spine 31: 1156-1162

\section{Social networks protect against cognitive impairment in Alzheimer's disease}

Studies have shown that the severity of cognitive impairment in Alzheimer's disease (AD) does not always correlate with the extent of disease pathology. It has been suggested that social interactions have a protective effect on cognitive function in people with the disease. Bennett et al. investigated the relationship between social network size, cognitive impairment and $A D$ pathology in elderly people with dementia who underwent annual clinical evaluation of cognitive function as part of the Rush Memory and Aging Project. Postmortem data were available for 89 participants, who had a mean social network size of 6.9 members.

Social network size was correlated positively with cognitive function but not with severity of $A D$ pathology. The relationship between the amount of $A D$ pathology and cognition, however, changed with the size of the social network such that the same amount of $A D$ pathology had less effect on cognitive test scores in persons with larger networks. The protective effect of social network size was most pronounced on the association of neurofibrillary tangles with semantic memory, but effects on the association of neurofibrillary tangles with working memory and episodic memory were also observed.

Although people with larger social networks have greater participation in cognitive, physical and social activities, involvement in such activities had no effect on the findings of the study, and controlling for depression had only a marginal effect. The authors suggest that aspects of social cognition involved in the development and maintenance of large social networks are associated with a cognitive reserve that compensates for neural degeneration and protects against cognitive impairment, even in advanced disease.

Original article Bennett DA et al. (2006) The effect of social networks on the relation between Alzheimer's disease pathology and level of cognitive function in old people: a longitudinal cohort study. Lancet Neurol 5: 406-412

\section{Nutritional supplement use in patients with Parkinson's disease}

Over the past decade, use of nutritional supplements has increased dramatically in the US, particularly among elderly people. There are few data on the use of nutritional supplements in patients with Parkinson's disease (PD), but adverse effects and harmful interactions with PD medications can occur. Wolfrath et al. have evaluated the use of nutritional supplements 